\title{
Thermo-Optical Property Degradation of ITO-Coated Aluminized Polyimide Thin Films Under VUV and Low-Energy Proton Radiation
}

\author{
MARTA DEMBSKA, THOMAS RENGER, and MACIEJ SZNAJDER \\ We studied thermo-optical property degradation of indium tin oxide (ITO)-coated aluminized \\ polyimide thin films under exposure to vacuum ultraviolet radiation and low-energy ( 3 and 5 \\ $\mathrm{keV}$ ) protons during ground tests using the Complex Irradiation Facility at the DLR site in \\ Bremen. Changes in solar absorption and thermal emission coefficients caused by the irradiation \\ were analyzed. We report a significant increase in solar absorptance of the samples irradiated by \\ protons. We also attempted to identify any defects on the surface of the samples. The study was \\ motivated by a unique opportunity that is provided by the Complex Irradiation Facility to study \\ the degradation effects induced by exposure to protons with an energy below $10 \mathrm{keV}$ and \\ short-wavelength light below $115 \mathrm{~nm}$.
}

https://doi.org/10.1007/s11661-020-05906-x

(C) The Author(s) 2020

\section{INTRODUCTION}

MATERIAls certified for use in space are characterized by their exceptional properties (for instance lightweight, resistance to ionizing radiation, multifunctional capabilities, self-healing capabilities and outstanding thermal stability) so that they can survive in an environment that combines, among others, ionizing radiation, extreme temperatures, micrometeorites, and deep vacuum. Many space applications require a coating that is applied to the surface of the material to protect it or change its properties. Both the materials and their coatings used for spacecraft applications must be easy to apply and should have low outgassing and stability in space environment.

However, despite the unique characteristics, space is a harsh environment for the materials used on spacecraft, especially on their exterior surfaces. Most of these materials exhibit some degree of degradation due to different external factors, including ultraviolet and particle damage. One of the crucial aspects in spacecraft design is the thermal control system whose function is to keep the temperature of spacecraft systems within their operating ranges. The absolute temperature of a given area of the spacecraft in interplanetary space far away

MARTA DEMBSKA is with Data Management and Analysis, DLR Institute of Data Science, Mälzerstraße 3, 07745 Jena, Germany. Contact e-mail:marta.dembska@dlr.de THOMAS RENGER and MACIEJ SZNAJDER are with the Mechanics and Thermal Systems, DLR Institute of Space Systems, Robert-Hooke-Str. 7, 28359 Bremen, Germany.

Manuscript submitted September 30, 2019.

Article published online July 19, 2020 from Earth's atmosphere is directly determined by the ratio between solar absorptance $\alpha_{s}$ and thermal emittance $\varepsilon_{t},{ }^{[1]}$ whereby $\alpha_{s}$ is defined as the fraction of incident solar radiant flux that is absorbed by a surface and $\varepsilon_{t}$ as the ratio of the radiant intensity of the surface to that which would be emitted from a black body radiator. ${ }^{[2]}$ Thus, any change in thermo-optical properties of the surface materials affect the operation of the thermal control system and - directly or indirectly - the space mission.

Thermal control of spacecraft is mainly achieved by isolating it from the harsh environment by means of the multi-layer insulation (MLI) that prevents the heat from the Sun entering the spacecraft and restricts the loss of internally generated heat from the spacecraft. Typically, MLI consists of many (from several to few tens) layers of usually lightweight low-emittance polyimide films that are aluminized on one or both sides, separated by low-conductance spacers that reduce the heat transfer between the aluminized polyimide that acts as a radiation shield. Passive thermal control is also achieved by the application of various coatings with known optical properties, for example, high emittance (to improve the heat radiation characteristics) or low emittance (to minimize the radiative coupling).

An example of materials widely used in space applications is Kapton, a polyimide film developed by DuPont $t^{\top M}$, that is stable across a wide range of temperatures $^{[3]}$ and used, among others, in flexible electronics and thermal blankets on spacecraft, satellites, and space instruments (see Reference 4 and references therein). When required, materials for spacecraft applications must be coated. One of the examples of a special purpose coating is indium tin oxide (ITO) that is often 
Table I. The Samples Parameters Before and After Exposure to VUV Light or Protons

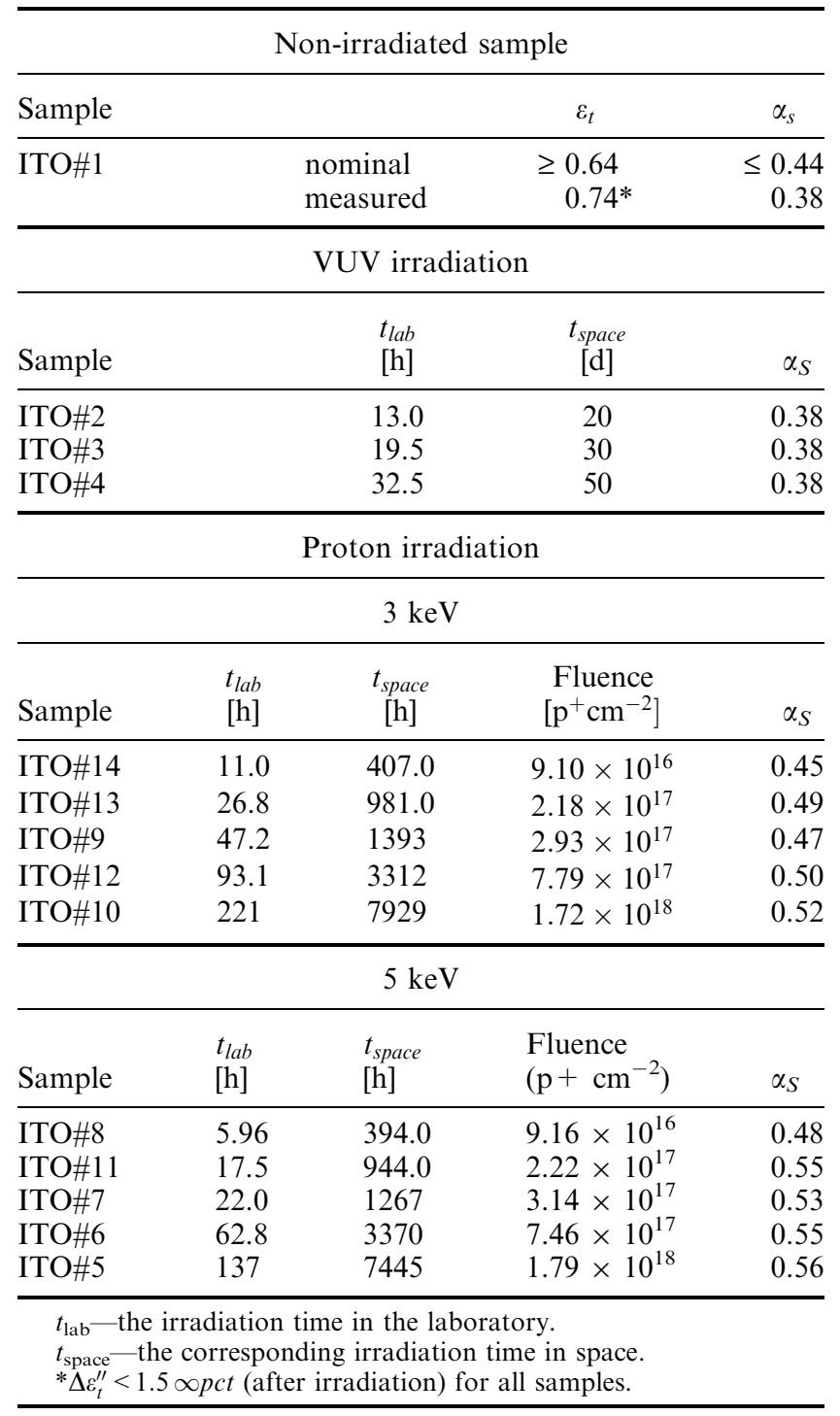

applied to the front surface of the second surface mirror (the second surface mirror consists of an optically smooth metallic surface and a protective layer so that the incident rays do not hit the high reflectance layer directly but pass through the protective layer before and after reflection). The ITO coating is used to drain static electricity that is induced on the outer surface whose charges can build potentials of 20,000 to 30,000 volts. The conductive coating is transparent, thus it does not significantly affect the emittance or solar absorptance of the second surface mirror.

Motivated by a very limited number of studies on low-energy proton radiation effects (see References 5, 6 and references therein) and vacuum ultraviolet (VUV) radiation in space materials, we used the Complex Irradiation Facility (CIF) that - to our knowledge - offers a unique system with sources of very low-energy protons (down to below $10 \mathrm{keV}$ ) and short-wavelength light (down to $40 \mathrm{~nm}$ ) to study the influence of

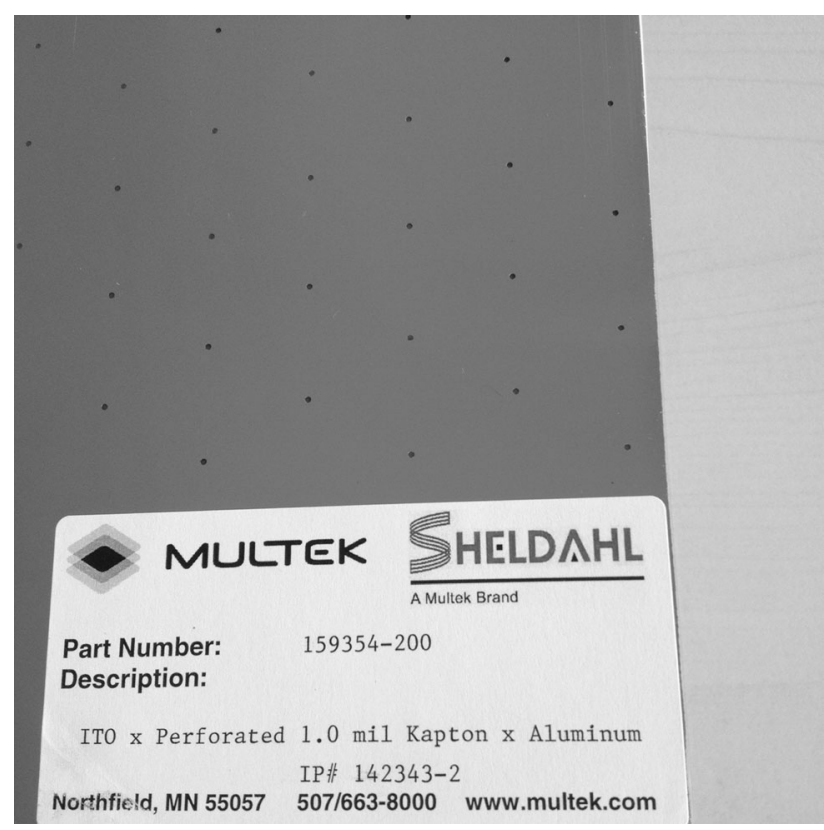

Fig. 1-A picture of a foil that was used in our experiments and its specification.

irradiation with the VUV light source and the bombardment of the probe with protons (for more details about the facility see Section II-A) on thermo-optical properties of the ITO-coated aluminized polyimide foils. The main goal of these studies was extrapolation of real processes following material exposure to space conditions. In this case, we extrapolated from degradation effects caused by irradiation of the probes in the CIF. It was achieved by measurements of changes in thermo-optical properties, $\alpha_{s}$ and $\varepsilon_{t}$, of the irradiated material after the exposure when compared to its state before the experiment. We also attempted to identify any defects induced by the irradiation.

\section{TEST DESCRIPTION}

In these studies, the Sheldahl brand material of ITO-coated aluminized polyimide films was investigated (see Figure 1). The structure of the films is as follows: a layer of a ITO coating was applied to the front side and a reflecting aluminum coating to the back side of a polyimide substrate (for more details visit the Red Book webpage.*) These films are often used as an outer layer in

*https://www.sheldahl.com/technology/thermal-control-materials/t he-red-book.

\section{MLI blankets.}

The polyimide layer is $1.0 \mathrm{mil}(25.4 \mu \mathrm{m})$ thick and the nominal aluminum coating thickness is $1000 \AA$ $(0.1 \mu \mathrm{m})$. The ITO coating has a standard target surface resistance of $5000 \Omega / \mathrm{sq}$. According to the manufacturer, it corresponds to a thickness of less than $100 \AA$ $(0.01 \mu \mathrm{m})$; thus, it should have no measurable impact 
on emittance and increases the solar absorptance by about 5 pct. The product was perforated prior to metalizing, which is a standard procedure to enhance its functionality. The samples were exposed to low-energy protons and VUV radiation in three sets of experiments using the CIF at a temperature of $300 \mathrm{~K}$.

\section{A. Complex Irradiation Facility}

\section{General description}

The Complex Irradiation Facility at the DLR Institute of Space Systems in Bremen (Germany), presented in Figure 2, is an instrument designed to study material surface property changes after exposure to corpuscular and electromagnetic radiation. Such studies are mandatory e.g., for a proper thermal design of satellite components exposed directly to radiation in a space environment. The facility is an Ultra High Vacuum system, equipped with two linear particle accelerators and three light sources. Both electrons and protons can be generated in an energy range from 2 to $100 \mathrm{keV}$. The maximum current of the particles can reach $100 \mu \mathrm{A}$ over a maximum area of $60 \times 60 \mathrm{~mm}$. All radiation sources can expose test specimens simultaneously, which presents a unique opportunity to study synergistic degradation effects on the materials under study. More importantly, each of the CIF sources can be used with an acceleration factor higher than one, which allows us to study how radiation with an intensity much higher than the equivalent of the Sun intensity influences degradation mechanisms.

\section{The particles irradiation system}

The corpuscular radiation of the CIF is achieved by use of two independent linear electron and proton accelerators. Both can irradiate specimens separately or simultaneously. The particle current can be set from 1 $\mathrm{nA}$ to $100 \mu \mathrm{A}$. Only one energy level (between 2 and 100 $\mathrm{keV}$ ) of the particles can be chosen; however, each particle source can irradiate the sample with different energy. Electrons are produced by heating up a $\mathrm{LaB}_{6}$ cathode. By changing the heater current and the Wehnelt voltage, one can control the electron current. Protons are produced by ionization of Hydrogen. $\mathrm{H}_{2}$ gas is pumped into a glass bulb where the ionization is
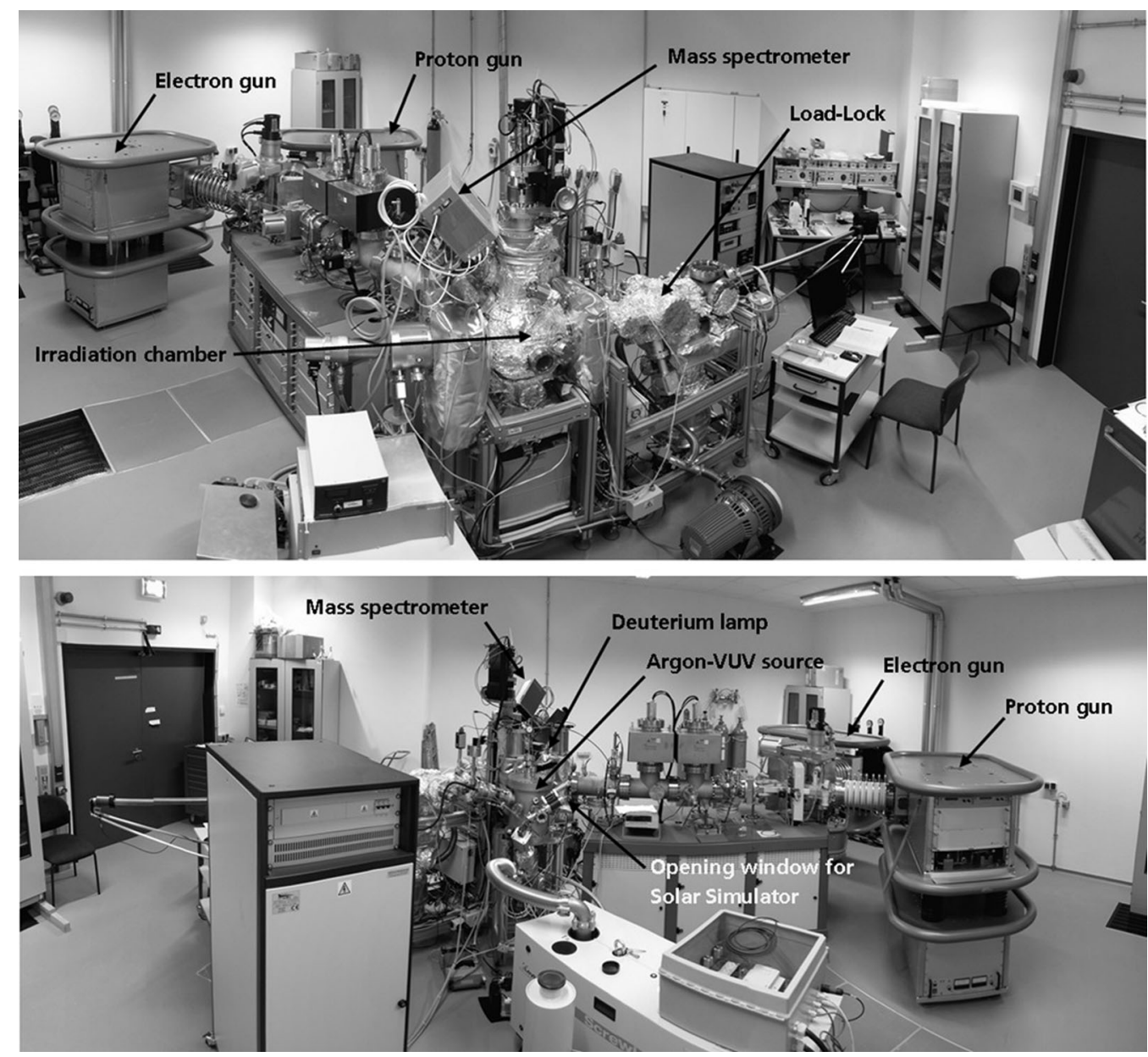

Fig. 2-An overview of the Complex Irradiation Facility (for more details of the instrument see Renger et al. ${ }^{[7]}$ ). 
achieved by radio frequency excitation. Protons, $\mathrm{H}^{2+}$, and $\mathrm{H}^{3+}$ ions are produced, which makes a mass selection necessary. It is accomplished by using a magnetic field to bend the particles beam. A careful selection of the field's strength enables to beam only the protons into the irradiation chamber, while the other ions strike the beamline wall.

The cross section of both beams has a circular shape and its diameter depends on the incident energy of the particles. The maximum diameter is approx. $4 \mathrm{~cm}$, while the minimum can be achieved by use of a retractable aperture plate with a drilled hole of approx. $1 \mathrm{~mm}$ in diameter, located in front of the irradiation chamber. The sample can be exposed to a maximum effective irradiation area of $6 \times 6 \mathrm{~cm}$. It is achieved by use of a beam sweep mechanism, which is accomplished by two pairs of plates, each with an opposite potential. Working together, they sweep the beam across the whole sample area.

\section{The light sources}

The CIF has three light sources: a Xenon lamp, a Deuterium lamp, and an Argon-VUV-source. All of the light sources can work simultaneously and cover a wide wavelength range from 40 to $2150 \mathrm{~nm}$. The Xenon lamp is calibrated on demand before each test, while the Deuterium lamp and the Argon source were calibrated at the Physikalisch-Technische Bundesanstalt (PTB) in Berlin. The Argon-VUV source that we used during our tests produces light by exciting an Argon (98.5 pct), Krypton (1 pct), and Helium (0.5 pct) gas mixture with a well-defined beam of $1 \mathrm{keV}$ electrons. The electrons pass through the gas flow located in the center of a vacuum chamber and between two nozzles. One pushes the gas with a defined flow rate, the second pulls the gas out of the chamber. The rest of the gas condensates on a cold baffle which surrounds the two nozzles. The gas atoms excited by the electrons drop their energy to the ground state and emit photons. Since the VUV source is continuously supplied with gas, which is then used to produce the light, the light intensity of the source remains relatively stable over time. The calibration campaign, the light spectrum for three different gas flows, together with identified emission lines, and the analysis of the source stability can be found in Reference [8]. The source produces VUV light with an intensity and wavelength range exceeding those produced by commonly used Deuterium lamps. These lamps have a wavenumber cut-off at around $115 \mathrm{~nm}$ which is related to internally used $\mathrm{MgF}_{2}$ or $\mathrm{LiF}_{2}$ windows. It means that, in the vast majority of degradation reports, materials are tested down to $115 \mathrm{~nm}$. Lower wavelength ranges are not considered. Hence, the VUV source presents a unique opportunity to examine the role of short-wavelength range light in the degradation of test materials.

\section{The sample holder}

Our standard sample holder is a frame consisting of two parts. The specimen is placed between the two and held by means of four screws. The maximum effective irradiation area is $60 \times 60 \mathrm{~mm}$. The frame is made up of stainless steel.

\section{Vacuum control}

Vacuum level in the empty irradiation chamber reaches a low $10^{-9}$ mbar range. The vacuum is achieved using a differential pumping system. After venting, the chamber is evacuated by the use of a scroll pump to a level of $10^{-3}$ mbar. When this pressure is reached, the turbo molecular pump is activated to lower the vacuum level to $10^{-8}$ mbar. The further step is achieved with an ion getter pump.

\section{Temperature control}

Temperature control is performed by means of a thermostat. Temperature is measured with two PT100 sensors. The first is located at the copper block, which is heated from behind by a set of halogen lamps and the second is inserted into the sample holder from the side while sliding it in to the sample station. The first is controlled by software, which-based on current temperature - turns on/off the lamps.

\section{Experiment procedure}

The sample material is first cut into a size of $65 \times 75$ $\mathrm{mm}$ and inserted between two frame plates and screwed together. Then it is inserted into the so-called load lock chamber of the CIF. It is mounted on the end of a magnetic arm. The lock is then closed and sealed, and the chamber is evacuated. Once the pressure in the chamber reaches the level of vacuum pressure in the irradiation chamber $\left(10^{-8} \mathrm{mbar}\right)$, a valve separating the two can be opened. The sample holder with the specimen is then transferred to the sample station where it can be exposed to radiation sources of the CIF.

\section{B. VUV Exposure}

Three samples of the ITO-coated aluminized polyimide thin films (ITO\#2, ITO\#3 and ITO\#4) were exposed to VUV light using the Argon VUV source. A gas flow of $1200 \mathrm{sccm}$ was chosen to reach the highest acceleration factor of 36.86 at $40 \mathrm{~nm}$. The experiments are equivalent to 20,30 , and 50 days, respectively (see Table I), in interplanetary space conditions (i.e., the region between celestial bodies that is far enough from Earth and other planets for the influence of the interplanetary plasma to be the dominant factor in materials degradation) at a distance of $1 \mathrm{AU}$ from the Sun at $40 \mathrm{~nm} \cdot{ }^{[8]}$

\section{Protons Exposure}

Ten samples of ITO-coated aluminized polyimide thin films were exposed to different proton fluence values (see Table I) using the linear proton accelerator with proton energies of $3 \mathrm{keV}$ (ITO\#9, ITO\#10, , ITO\#12, ITO\#13 and ITO\#14) and $5 \mathrm{keV}$ (ITO\#5, ITO\#6, ITO\#7, ITO\#8, ITO\#11). The average fluence was $6.33 \times 10^{17}$ $\mathrm{p}^{+} \mathrm{cm}^{-2}$ for an energy of $5 \mathrm{keV}$ and $6.20 \times 10^{17} \mathrm{p}^{+} \mathrm{cm}^{-2}$ for an energy of $3 \mathrm{keV}$. The exposure times, lasting from hours to days, were chosen to obtain five equivalent fluence values for both energies. 
Taking into consideration $\alpha_{s} / \varepsilon_{t}=0.51$, these experiments are equivalent to $10,24,34,80$, and 193 hours for an energy of $5 \mathrm{keV}$ and 10, 24, 32 hours, 84, and 185 minutes for an energy of $3 \mathrm{keV}$ in interplanetary space conditions at a distance of $0.89 \mathrm{AU}$ from the Sun. ${ }^{[9-11]}$

The Stopping and Range of Ions in Matter (SRIM) package $^{[12]}$ and the PSTAR ${ }^{[13]}$ software were used to estimate the penetration depth of protons with energies of 3 and $5 \mathrm{keV}$ in a thin film of a structure such as the ones used in our studies. Assuming that the ITO thickness is less than $100 \AA$, protons pass through the first layer and stop in the polyimide.

\section{Optical Measurements}

The solar absorption coefficient $\alpha_{s}$ and the thermal emission coefficient $\varepsilon_{t}$ of a material used in space applications are critical parameters in determining its thermo-optical properties. Absorbed solar radiation is typically the predominant external heat input to the spacecraft. Thermal emittance describes the rate at which heat leaves the spacecraft. Degradation of $\alpha_{S}$ and/ or $\varepsilon_{t}$ causes changes to the temperature of outer components which can influence the spacecraft temperature. Therefore, it is necessary to conduct laboratory testing on materials before certifying them for use in space.

In these studies, both coefficients were calculated for our samples before and after irradiation, based on spectral reflectance measurements as follows ${ }^{[2]}$

$$
\alpha_{s}=1-R_{s}=1-\frac{\int_{250 \mathrm{~nm}}^{2500 \mathrm{~nm}} R(\lambda) S(\lambda) d \lambda}{\int_{250 \mathrm{~nm}}^{2500 \mathrm{~nm}} S(\lambda) d \lambda},
$$

and

$$
\varepsilon_{t}=\frac{\int_{3 \mu m}^{20 \mu m} A(\lambda) E(\lambda) d \lambda}{\int_{3 \mu m}^{20 \mu m} E(\lambda) d \lambda},
$$

where $R(\lambda)$ is the spectral reflectance after 100 pct reference correction, $S(\lambda)$ is the spectral solar irradiance, $A(\lambda)=1-R(\lambda)$, and $E(\lambda)$ is the black body emittance spectrum at $300 \mathrm{~K}$.

We used a VERTEX 80v vacuum FTIR spectrometer from Bruker, equipped with integrating spheres with a sample holder on the sidewall. The optical measurements were perfomed ex situ. Reflectivity was compared to the known mirror standards. The uncertainties were calculated as the standard deviation from series of repeated spectral reflectance measurements in a given range.

\section{RESULTS AND DISCUSSION}

\section{A. Overview of Sample Degradation}

Each sample was carefully investigated with the naked eye before and after exposure. The samples exposed to VUV radiation had their whole surface irradiated. We report no visible degradation of the samples. A limited area of each sample was irradiated by low-energy protons. We report a visible color change caused by the proton bombardments (see Figure 3).

We also examined the samples with the field-emission and atomic force microscopes (FEM and AFM) to identify any defects induced by the irradiation. In both cases, there was no apparent change to the surface structure that could be directly linked to degradation effect caused by the VUV light or the proton bombardment.

\section{B. Spectral Reflectance Change}

Figures 4 and 5 show changes in spectral reflectance of the samples exposed to protons of an energy of $3 \mathrm{keV}$ or $5 \mathrm{keV}$ respectively. Low-energy protons clearly cause a general decrease of the spectral reflectance. The change is especially significant in the visible and infrared regions of the electromagnetic spectrum. For both energies, the increase in proton fluence up to above $5 \times 10^{19} \mathrm{p}^{+} \mathrm{cm}^{-2}$ (ITO\#14 and ITO\#8 respectively) results in a strong reduction of the reflectance. Then, with further rise in proton fluence, at first, the reflectance shows a temporary reverse of the reduction trend to then maintain a less intense but steady decrease.

\section{Thermo-Optical Properties}

Table I presents $\alpha_{s}$ for all samples. The change in the thermal emission coefficient after irradiation was less than 1.5 pct. Thus, only the value for a non-irradiated material is shown.

\section{VUV irradiation}

A first noticeable change in $\alpha_{S}$ was observed after an equivalent of 50 days in space at distance of a $1 \mathrm{AU}$ from the Sun at $40 \mathrm{~nm}$. These results suggest a VUV radiation hardness of this material at very short wavelengths for short-term exposures.

\section{Proton irradiation}

Low-energy protons caused a significant increase in the solar absorption coefficient when compared to the non-irradiated material. Figure 6 presents $\alpha_{s}$ change with increasing protons fluence for both energies of $3 \mathrm{keV}$ and $5 \mathrm{keV}$. The increase in the solar absorption coefficient varies from 18 pct for ITO\#8 to 36 pct for ITO\#5 at $3 \mathrm{keV}$ and from 28 pet for ITO\#8 to 49 pct for ITO\#5 at $5 \mathrm{keV}$ when compared to a non-irradiated sample.

Additionally, there is a nearly perfect scaling between the solar absorption coefficients for equivalent fluence values at $3 \mathrm{keV}$ and $5 \mathrm{keV}$ as follows: 

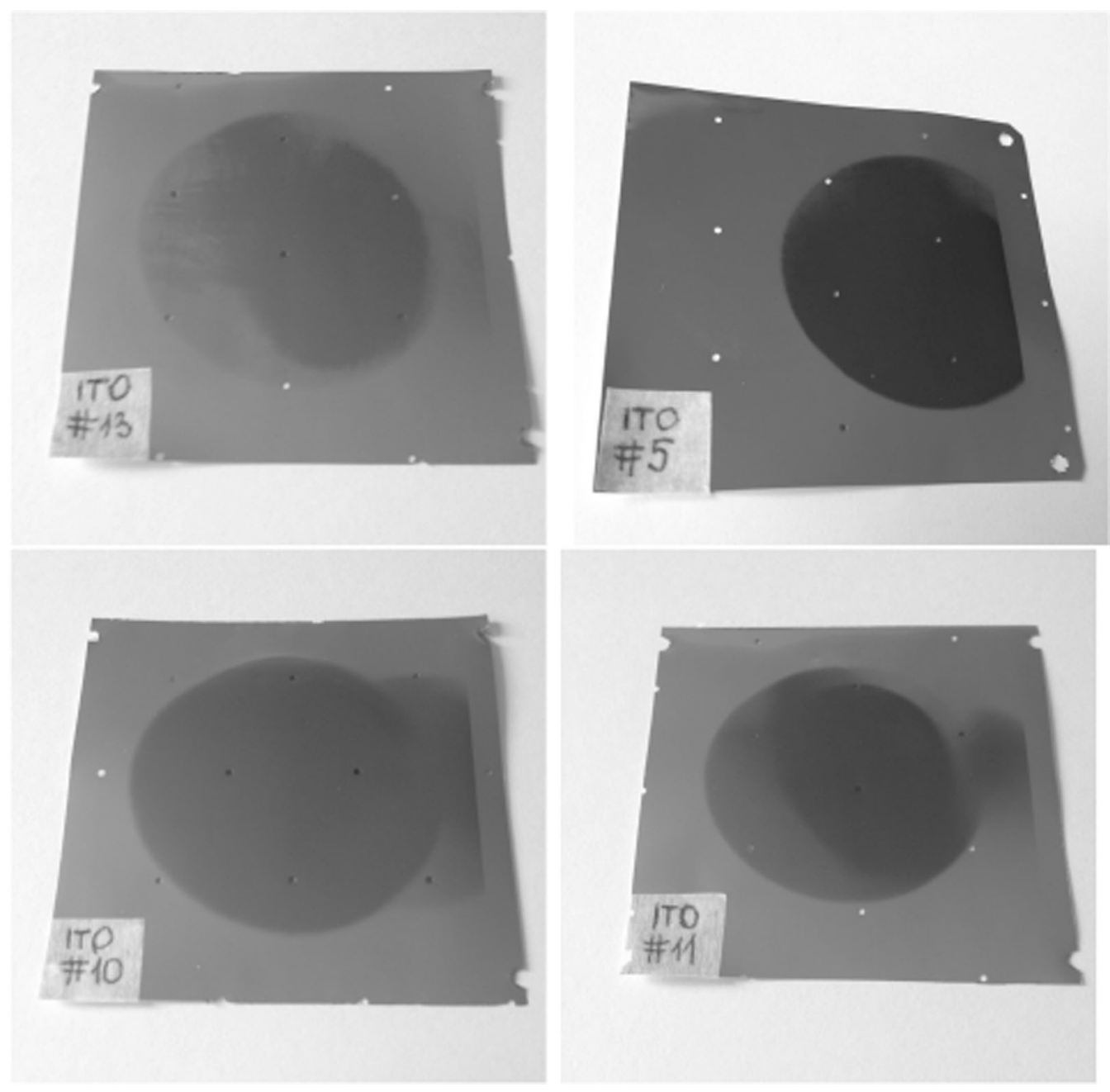

Fig. 3-ITO-coated aluminized polyimide thin films, samples after exposure to proton energies of $3 \mathrm{keV}$ (ITO\#13 and ITO\#10) and $5 \mathrm{keV}$ (ITO\#5 and ITO\#11), respectively. The darker areas were irradiated and show a visible change in color when compared to the non-irradiated part of the sample. For more details of the setup and parameters of the experiments, see text and Table I.

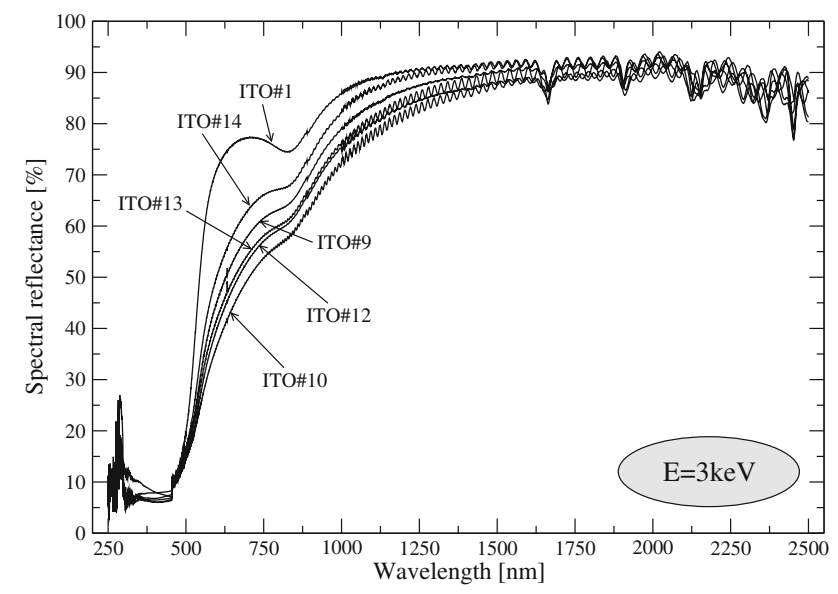

Fig. 4 - Spectral reflectance of the investigated samples of aluminized Kapton film with an ITO coating after $3 \mathrm{keV}$ protons irradiation in comparison to the non-irradiated sample. See Table I for the values of the fluence and the corresponding irradiation time in space.

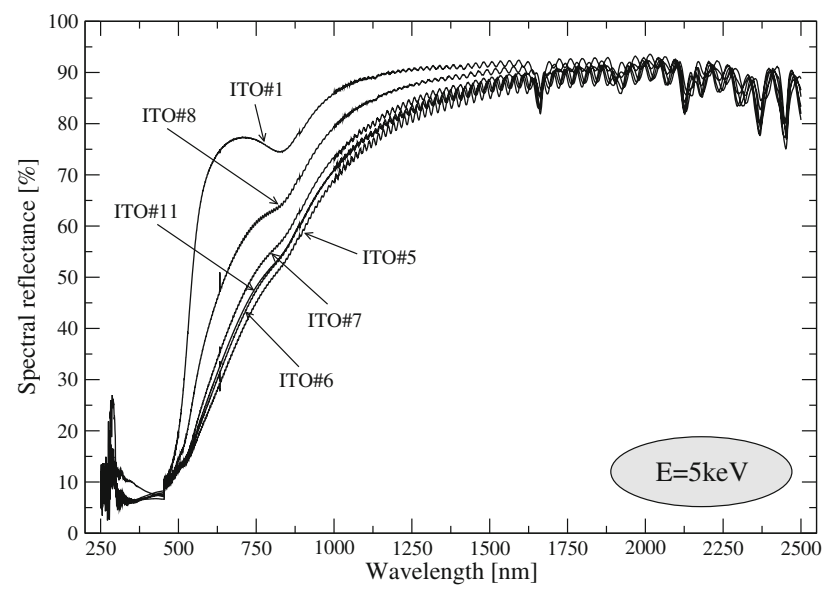

Fig. 5- Spectral reflectance of the investigated samples of aluminized Kapton film with an ITO coating after $5 \mathrm{keV}$ protons irradiation in comparison to the non-irradiated sample. See Table I for the values of the fluence and the corresponding irradiation time in space. 


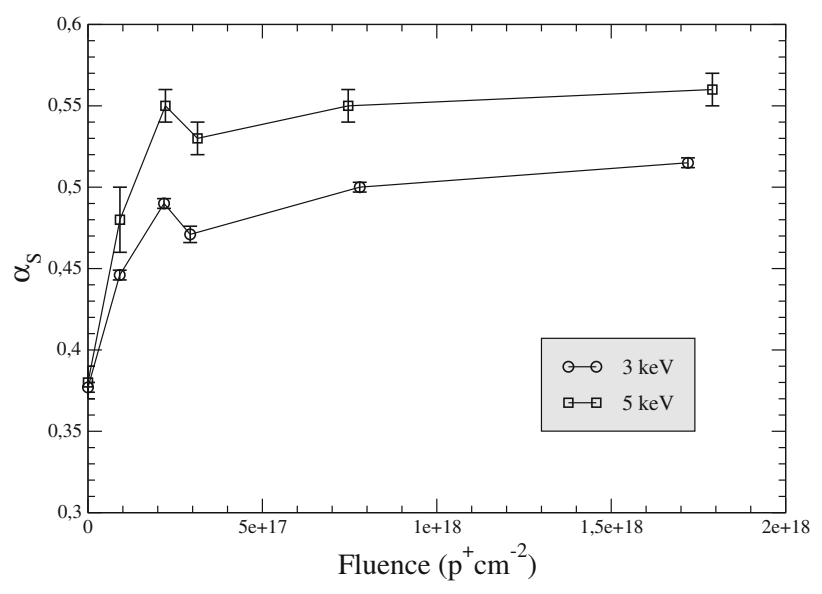

Fig. 6- Solar absorption coefficient of the samples after an exposure to five different proton fluence values for a proton energy of 3 and 5 $\mathrm{keV}$.

$$
\alpha_{s}^{5 k e V}=1.1 \times \alpha_{s}^{3 k e V} .
$$

Degradation in solar absorption, as expected from the reflectance spectra, generally increases when the fluence value rises. We also observe a temporary decrease of $\alpha_{s}$ (ITO\#9 and ITO\#7 respectively). The increase is steeper for the first two fluence values than for the two last ones.

\section{DISCUSSION}

\section{A. Ultraviolet}

MLI was reported by Sharma and Sridhara ${ }^{[1]}$ to degrade after UV, sequential UV, and charged particle irradiation exposure. A significant increase of $\alpha_{s}$ and only a slight rise in $\varepsilon_{t}$ were observed. However, UV sources available in most facilities used for material tests do not allow irradiation with wavelengths shorter than approx. $115 \mathrm{~nm}$ (an intensity cut-off of a common Deuterium lamp). Despite the fact that VUV radiation (10 to $200 \mathrm{~nm}$ ) constitutes only about $0.1 \mathrm{pct}$ of the near ultraviolet (NUV) irradiation intensity, it can still significantly contribute towards material degradation. The energy of a single photon in VUV range is high enough to cause photoionization and photodissociation effects that cannot be generated by NUV photons. Thus, one would expect a degradation of materials exposed to VUV radiation, especially for longer periods of time. Our results show no degradation in thermo-optical parameters of the films at $40 \mathrm{~nm}$ for exposures that correspond to fewer than 50 days in space at a distance of 1 AU from the Sun.

\section{B. Protons}

Li et al. ${ }^{[5]}$ reported changes in spectral reflectance of aluminized Kapton under proton irradiation with the energy of $90 \mathrm{keV}$. However, they observed a monotonic decrease of spectral reflectance in the 500 to $1200 \mathrm{~nm}$ wavelength region with increasing proton fluence. The same samples were reported to exhibit an increase of surface roughness that was examined by AFM. ${ }^{[6]}$ Our results show a temporary reverse of the growth trend of degradation in the $5-7 \times 10^{19} \mathrm{p}^{+} \mathrm{cm}^{-2}$ fluence range. The surface of our samples was not changed in a similar way, which could have been a protective effect of the ITO coating.

\section{Further Research}

Further studies of degradation of the material caused by the VUV radiation would require much longer exposures of the films to get more reliable information. As discussed by Li et al. ${ }^{[5]}$ forming new chromophores and auxochromes could cause the visible color change of the samples induced by protons and the increase in absorbance in the visible region. However, we report the temporary healing-like effect between samples ITO\#13 and ITO\#9 for a proton energy of $3 \mathrm{keV}$, and ITO\#8 and ITO\#11 for a proton energy of $5 \mathrm{keV}$ (see Figure 6), despite increasing the fluence. Thus, to explain the observed effect in a quantitative and qualitative manner, further research, both experimental and theoretical, is required. We intend to perform further irradiation of aluminized Kapton thin films with low-energy protons. Additionally, since Ding et al. ${ }^{[14]}$ reported an increase in solar absorptance of ITO-coated aluminized Kapton under electron irradiation, an investigation of synergic influence of both protons and electrons on aluminized Kapton thin films would contribute to our understanding of the changes in internal structure and surface properties of the material when exposed to space conditions. Other aspects worth addressing are a possible influence of the temperature of the sample and the acceleration factor.

\section{CONCLUSION}

Testing degradation effects caused by interplanetary conditions is especially important for any planned space mission as there is no sample available that had been exposed to such an environment and then brought back to Earth. ${ }^{[15]}$ Studies on Kapton degradation due to its diverse applications (e.g., MLI blankets and solar sail membranes) attracts the attention, both from the theoretical and experimental point of view, of space scientists and engineers.

The results of exposure of ITO-coated aluminized polyimide thin films to VUV radiation in our facility suggest a VUV radiation hardness of this material at very short wavelengths for short-term exposures. According to the presented results, low-energy protons cause a significant rise in the solar absorption coefficient of ITO-coated aluminized polyimide thin films.

Radiation hardness of materials during a whole space mission is highly desirable and often crucial for the accomplishment of these projects. The findings presented here constitute the first block of a planned set of experiments to study degradation effects caused by different environmental conditions induced in the materials that are used in space. 
The main motivation for using the Complex Irradiation Facility is to extend the existing results of ground experiments with a unique proton energy range (down to below $10 \mathrm{keV}$ ) and short-wavelength light (down to $40 \mathrm{~nm})$.

\section{ACKNOWLEDGMENTS}

Open Access funding provided by Projekt DEAL.

\section{OPEN ACCESS}

This article is licensed under a Creative Commons Attribution 4.0 International License, which permits use, sharing, adaptation, distribution and reproduction in any medium or format, as long as you give appropriate credit to the original author(s) and the source, provide a link to the Creative Commons licence, and indicate if changes were made. The images or other third party material in this article are included in the article's Creative Commons licence, unless indicated otherwise in a credit line to the material. If material is not included in the article's Creative Commons licence and your intended use is not permitted by statutory regulation or exceeds the permitted use, you will need to obtain permission directly from the copyright holder. To view a copy of this licence, visit http://creat ivecommons.org/licenses/by/4.0/.

\section{REFERENCES}

1. A.K. Sharma and N. Sridhara: Adv. Space Res., 2012, vol. 50, pp. 719-22.
2. ECSS-Q-ST-70-09C. Space product assurance. Measurements of thermo-optical properties of thermal control materials (European Space Agency, 2018), http://esmat.esa.int/ecss-q-st-70-09c.pdf, Accessed 15 May 2019.

3. R. Mishra, S.P. Tripathy, K.K. Dwivedi, D.T. Khathing, S. Ghosh, and D. Fink: Radiat. Meas., 2003, vol. 36, pp. 719-22.

4. E.A. Plis, D.P. Engelhart, R. Cooper, W.R. Johnston, D. Ferguson, and R. Hoffmann: Appl. Sci., 2019, vol. 9, art. no. 1999.

5. R. Li, C. Li, S. He, M. Di, and D. Yang: Radiat. Phys. Chem., 2007, vol. 76, pp. 1200-04.

6. R. Li, C. Li, S. He, M. Di, and D. Yang: Radiat. Phys. Chem., 2008, vol. 77, pp. 482-89.

7. T. Renger, M. Sznajder, A. Witzke, and U. Geppert: J. Mater. Sci. Eng. A, 2014, vol. 4, pp. 1-9.

8. M. Sznajder, T. Renger, A. Witzke, U. Geppert, and R. Thornagel: Adv. Space Res., 2013, vol. 52, pp. 1993-2005.

9. M. Sznajder, U. Geppert, Proceedings of the 13th European Conference on Spacecraft Structures (ESA Special Publication, Braunschweig, Germany, Materials \& Environmental Testing, 2014), pp. 1-8.

10. M. Sznajder, U. Geppert, and M. Dudek: Adv. Space Res., 2015, vol. 56, pp. 71-84.

11. M. Sznajder, U. Geppert, and M.R. Dudek: NPJ Mater. Degrad., 2018, vol. 2, art. no. 3 .

12. J.F. Ziegler and J.P. Biersack: Treatise on Heavy-Ion Science, Springer, Boston, 1985, pp. 93-129.

13. M.J. Berger, J.S. Coursey, M.A. Zucker, J. Chang, ESTAR, PSTAR, and ASTAR: Computer programs for calculating stopping-power and range tables for electrons, protons, and helium ions (version 1.2.3), (National Institute of Standards and Technology, Gaithersburg, MD, 2005), http://physics.nist.gov/Star, Accessed 8 July 2019.

14. Y. Ding, W. Feng, D. Yan, Degradation of optical-properties of thermal control coatings under space low energy electrons (Proceedings of the 11th ISMSE: International Symposium on Materials in Space Environment, 2009) http://esmat.esa.int/materials_ news/isme09/pdf/3-Ground/Poster pct20Ground pct20Testing pc t20- pct20 pct20Ding.pdf. Accessed 17 May 2019.

15. M. Sznajder, U. Geppert, Proceedings of the 14th European Conference on Spacecraft Structures, Materials and Environmental Testing, Toulouse, France, 18 (2016).

Publisher's Note Springer Nature remains neutral with regard to jurisdictional claims in published maps and institutional affiliations. 